\title{
Functional information and entropy in living systems
}

\author{
A. C. McIntosh \\ Energy and Resources Research Institute, University of Leeds, Leeds, UK
}

\begin{abstract}
In any living system one quickly becomes aware of the extraordinary complexity that so organises the chemical proteins at the biochemical level as to effectively build digital machinery which for many years, since the discovery by Crick and Watson of DNA, has been the goal of modern software engineers to emulate. The functional complexity of these systems is clearly heavily dependent on the material environment in which such a system is operating and indeed uses all the same chemical and physical laws that are used to such good effect by any man made machines. What though are the laws that such organisation must inherently obey for natural systems? Can one quantify the organisational structure that sits on top of the matter and energy in any real system?

In this paper, the author will consider the fundamental aspects of entropy and the second law of thermodynamics applied first of all in the traditional definitions used in heat and chemical systems. Then analogous representations of 'logical entropy' will be discussed where for a number of years many scientists (such as Prigogine) have been attempting to simulate in a rational way the idea of functional complexity. Prigogine's work has primarily been seeking to express self organisation in terms of non-equilibrium thermodynamics and the term 'Prigogine entropy' has thus been introduced. Allied closely to this is the concept of the definition of information which must go beyond the simple recipe of Shannon's Theory, that essentially only deals with the transmission of existing data. The main issue at stake in any discussions of functional complexity is arriving at a logical approach to describing the possible states of the system, and secondly to establishing a valid proportionality constant that is analogous to the Boltzmann constant of traditional thermodynamics. In this paper we discuss how the laws of thermodynamics can be understood in terms of the possible information content of molecules. We build on the concept of information transfer and the notion of 'logical entropy', to considering the application of the laws of thermodynamics to non-equilibrium chemistry. This then concerns the basic definition of how information is defined and connected to the fundamental laws of thermodynamics. Although the paper may raise more questions than answers, the aim will be to at least move further towards a rigorous scientific treatment of the whole concept of organisation and system structure by seeking parallel (logical) laws of complexity in system states to the well known laws of thermodynamics.
\end{abstract}




\section{Introduction}

The defining of information is a key issue in the origins debate, since terms such as 'advance' and 'simple to complex' have little direct meaning at the biochemical level. Intuitive reasoning presupposes the worldview of such statements, and the discussions on origins are fundamentally to do with worldviews. Consequently the biochemical arguments are always going to be a vital battleground because of the root issues at stake.

Claude Shannon in 1948 [3] introduced the basis for the definition of the unit of information content. He argued that any logical process can be reduced to a series of either/or decisions (called in mathematics Booleian Algebra). Each decision can be represented by a 1 or 0 , represented in computer hardware terms by whether a microcircuit is 'on' or 'off' respectively. This unit is termed a 'bit' of information, and as complication increases it is more convenient to use the unit of a byte ( 8 bits). Thus any system and its information content can now be quantified in terms of this unit of information. Dawkins referring to the Shannon concepts in an essay entitled 'The Information Challenge' [4] made the following statement which is quoted in full, since it lies right at the heart of the thesis held by most evolutionary biologists that information increase is possible by natural selection operating on successive mutations :

"Let me turn, finally to another way of looking at whether the information content of genomes increases evolution. We now switch from the broad sweep of evolutionary history to the minutiae of natural selection. Natural selection itself, when you think about it, is a narrowing down from a wide initial field of possible alternatives, to the narrower field of the alternatives actually chosen. Random genetic error (mutation), sexual recombination and migratory mixing all provide a wide field of genetic variation: the available alternatives. Mutation is not an increase in true information content, rather the reverse, for mutation in the Shannon analogy, contributes to increasing the prior uncertainty. But now we come to natural selection, which reduces the 'prior uncertainty' and therefore, in Shannon's sense, contributes information to the gene pool. In every generation, natural selection removes the less successful genes from the gene pool, so the remaining gene pool is a narrower subset. The narrowing is non-random, in the direction of improvement, where improvement is defined, in the Darwinian way, as improvement in fitness to survive and reproduce. Of course the total range of variation is topped up again in every generation by new mutation and other kinds of variation. But it still remains true that natural selection is a narrowing down from an initially wider field of possibilities, including unsuccessful ones, to a narrower field of successful ones. This is analogous to the definition of information with which we began: information is what enables the narrowing down from prior uncertainty (the initial range of possibilities) to later certainty (the 'successful' choice among prior probabilities). According to this analogy, natural selection is by definition a 
process whereby information is fed into the gene pool of the next generation."

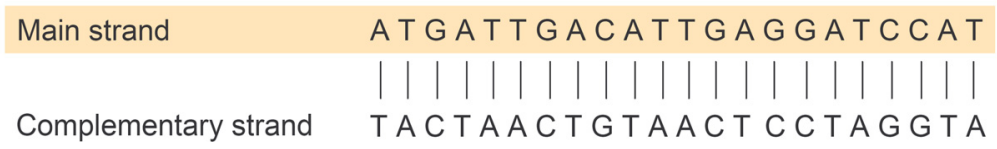

Figure 1: $\quad$ Sample genetic code with complementary strands.

This important paragraph shows the dilemma that faces an approach which only considers matter and energy without information (the 'bottom up' approach). There is an admission of the need for new information to counter the narrowing effect of natural selection as usually defined. This definition is simply that natural selection is the favourable advantage of random mutations in one generation making that alteration more likely to survive and be thus more prolific in the next generation. The narrowing effect is that the number of options to choose from is reduced since once the selection is made, the original gene pool is reduced. The answer suggested here is to alter the definition of natural selection (see last sentence of quote) and to further propose the topping up of the gene pool by the very mutations themselves. The formidable obstacles to this proposition lie on a macroscopic level in the very nature of DNA (see fig. 1) which has been shown to have the immense capability of in situ repair work. For example there are enzymes which are specifically assigned to nucleotide excision repair - they recognise wrongly paired bases in the DNA nucleotides (Adenine (A), Thymine (T), Cytosine (C) and Guanine (G)) connecting the two deoxyribose sugar-phosphate strands. This means that mutations are generally corrected (see for example the papers by Jackson [5] and de Laat et al [6]), so that even if speciation does occur due to slight modifications and adaptations of the phylogeny, any serious departures in the genetic information would be acted against by the DNA's own repair factory. Mutations do not increase information content - rather the reverse is true. The flightless Galapagos Cormorant is a classic example. Evidently repair by the above techniques was not possible, and the genetic defect has persisted, such that information has certainly been lost, and the gene pool (in that case irrevocably) reduced. At the very least Dawkins' assertion at the end is misleading, for it suggests there is a natural source of new information which experimental observation denies. Natural selection cannot be redefined and is not the handmaid of macro evolution.

However there is a more fundamental issue. At the molecular level, the laws of thermodynamics do not permit step changes in the biochemical machinery set up for a particular function performed by the cells of living organisms. That is any random mutations always have the effect of increasing the disorder (or what we will shortly define as logical entropy) of any particular system, and consequently decreasing the information content. What is evident is that the initial information content rather than being small must in fact be large, and is in fact vital for any process to work to begin with. The issue of functional complexity and information is considered exhaustively by Meyer [7] who argues that the neo-Darwinist model cannot explain all the appearances of design in 
biology. Even within the neo-Darwinist camp the evidence of convergence (similarity) in the suggested evolutionary development of disparate phylogeny has caused some writers [8] to consider 'channelling' of evolution. Such thinking is a tacit admission of a teleological influence. That information does not increase by random changes (contrary to Dawkins' assertion) is evident when we consider in the following section, the logical entropy of a biochemical system.

\section{The second law of thermodynamics}

A succinct statement of the second law is "The amount of energy available for useful work in a given system is decreasing. The entropy (dissipated useful energy per degree Kelvin) is always increasing."

Examples of this principle abound. Heat always flows from hot to cold. In the process it can be made to do work but always some energy will be lost to the environment, and that energy cannot be retrieved. Water flows downhill and loses potential energy which is changed into kinetic energy. This can again be made to do work (as in a hydroelectric power plant). However some energy will be lost such that if one was to use all the energy generated to pump the same water back up to its source, it would not reach the same level. The difference of original potential energy to that corresponding to the new level, divided by the temperature (which in that case is virtually constant) is the entropy of the system. Such a measure will always give an entropy gain.

There is no known system where this law does not apply. The fact that the entropy of a given closed system increases, effectively brings with it an inevitable decline in usefulness of all systems. The phrase 'arrow of time' is often used to describe this since the second law brings in the concept of nonreversibility of all real systems.

\subsection{The second law and open systems}

In that the second law of inevitable entropy increase applies to a closed system, some have maintained that with an open system one could have entropy decreasing in one area while the overall entropy of the two systems together (closed) is increasing. An illustration would be of two ice boxes A and B (see fig. 2) where there is an allowance for small contact between them but with (perfect) insulation round the rest of the cube $A$ and poor insulation round cube B. Systems A and B are both then open systems, as is the system A and B together (referred to as $\mathrm{A}+\mathrm{B}$ ), but system $\mathrm{A}$ and $\mathrm{B}$ with the surrounding region 1 , (that is the complete system) is closed. The entropy of the overall complete system then must increase with time. That is there will eventually be equilibrium throughout every region. Suppose we start with Temperature $T_{1}$ appreciably hotter than $T_{A}$ and $T_{B}$. Thus for instance we could have $T_{1}=100^{\circ} \mathrm{C}$ and $T_{A}$ and $T_{B}$ both at $-10^{\circ} \mathrm{C}$. Initially as time progresses the original equal temperatures $T_{A}$ and $\mathrm{T}_{\mathrm{B}}$ become different. $\mathrm{T}_{\mathrm{A}}$ will stay close to the original $-10^{\circ} \mathrm{C}$, but $\mathrm{T}_{\mathrm{B}}$ will begin to move to a higher value (say $+5^{\circ} \mathrm{C}$ ) due to there being good conduction of heat into ice box B (as against the insulated ice box A). Now consider system 
$\mathrm{A}$ and $\mathrm{B}$ together $(\mathrm{A}+\mathrm{B})$. One now has an open system with decreasing entropy, in that useable energy transfer between the two ice boxes is possible, and work can be achieved where before in that system, treated in isolation, none was possible. However one notes two things. First that this is possible only for a finite time - eventually the temperature difference will reach a maximum (when $T_{B}$ gets close to $T_{1}$ ) and at this point system $A+B$ will have a minimum entropy condition. After this system $\mathrm{A}+\mathrm{B}$ will then experience a rising entropy condition. Notice also that the initial conditions (different insulation levels) are important for it to be possible to achieve a low entropy condition local to system $\mathrm{A}+\mathrm{B}$. Effectively one has an elementary 'machine' which is making use of the nonhomogeneous temperature across the complete system. This demonstrates the reality of how the second law applies in open systems, and that extra energy from outside is no use unless there is a machine (i.e. teleonomy / information) available.

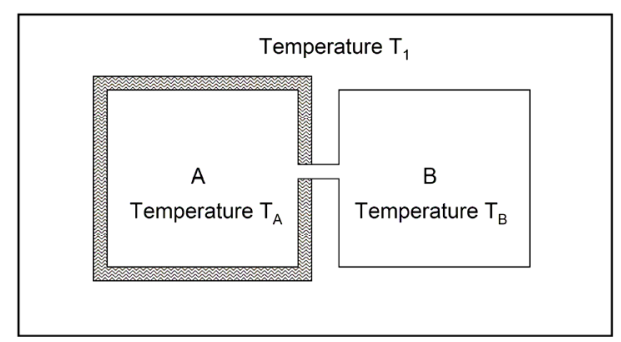

Figure 2: $\quad$ Open system A and B.

\subsection{Thermodynamic entropy and logical entropy}

A connection can also be made between entropy and disorganisation or disorder. The first to formalise this use of the concept of entropy was the Austrian physicist Ludwig Boltzmann.

Klyce [9] in a useful article, introduces the concept of logical entropy as follows. As the laws of thermodynamics were investigated in the latter part of the nineteenth century, it was evident that the second law implied there was a preferred direction in time even at the molecular level, which seemed to contradict the growing physical understanding of the laws of physics applied to molecular collisions, which indicated here there was no preferred direction in time - an elastic collision between molecules would look the same going forward or backward. In the 1880s and 1890s, Boltzmann used molecules of gas as a model, along with the laws of probability, to show that there was no real conflict. The model showed that heat, no matter how it was introduced, would soon become evenly diffused throughout the gas, as the second law required.

The cleverness of Boltzmann's ideas however was that the model could also be used to show that two different kinds of gases would become thoroughly mixed even though the temperature of each gas may in fact be the same. Thus an analogy is really being made between the diffusion of heat and the diffusion of 
two gases. The parallel between disorganisation and diffusion across basic distinct states was thus made. Quoting Klyce [9]

"The reasoning used for mixing is very similar to that for the diffusion of heat, but there is an important difference. In the diffusion of heat, the entropy increase can be measured with the ratio of physical units, joules per degree. In the mixing of two kinds of gases already at the same temperature, if no heat is exchanged, the ratio of joules per degree - thermodynamic entropy - is irrelevant. The mixing process is related to the diffusion of heat only by analogy. Nevertheless, Boltzmann used a factor, now called Boltzmann's constant, to attach physical units to the latter situation. Now the word entropy has come to be applied to the mechanical mixing process, too. (Of course, Boltzmann's constant has a legitimate use - it relates the average kinetic energy of a molecule to its temperature.)"

To gain understanding of this type of model of logical entropy we illustrate by following the example of the entropy of a gas using the Boltzmann approach.

\subsection{Entropy of a gas - an example of ordered states}

The entropy of a gas is given by

$$
s=-\frac{k}{W} \sum_{i} f_{i} \ln f_{i},
$$

where $i$ : tabulates the state $i$ This is usually a speed. Thus $i=10$ could represent the state of molecules moving in the $x$ - direction at say speed $10 \mathrm{~m} \mathrm{~s}^{-1}$. There can be negative $i$ 's as well. $W$ is the molecular weight $\left(\mathrm{kg} \mathrm{mol}^{-1}\right)$ and $k$ is Boltzmann's constant $\left(k=1.3805 \times 10^{-23} \mathrm{~J} \mathrm{~mol}^{-1} \mathrm{~K}^{-1}\right)$, so that the entropy is in specific terms (energy per unit mass per degree, $\mathrm{J} \mathrm{kg}^{-1} \mathrm{~K}^{-1}$ )

$f_{i}$ is the fraction of the parts (i.e. of the molecules) which are in state $i$ - i.e. moving at a certain speed. The sum $\sum$ will add the terms $f_{i} \ln f_{i}$ for all the parts (speeds). The $f_{i}$ 's are fractions between 0 and 1 , so that the $\log$ function $(\ln \equiv$ $\log _{\mathrm{e}}$ ) will be negative and $S$ will thus be positive.

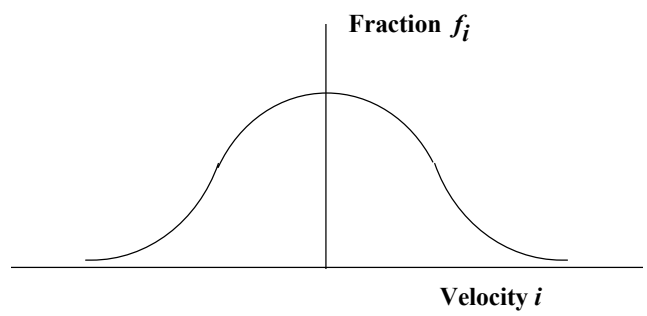

Figure 3: A particular non-equilibrium state (normally distributed).

Suppose all the molecules are moving at $10 \mathrm{~m} \mathrm{~s}^{-1}$, then all of the parts of the system would be in state $i=10$, so $f_{10}$ would be 1 with the rest of the $f_{i}$ 's at zero. Now for $f_{i}=0$ or 1 , then $f_{i} \ln f_{i}=0$. For a particular state of non-equilibrium, there is roughly a normal distribution of possible states with a mode near one state (see fig. 3) so that with the maximum $f_{\mathrm{i}}$ being less than unity, the $\log _{\mathrm{e}}$ of all the $f_{\mathrm{i}}$ 's is 
negative, and therefore $\sum f_{\mathrm{i}} \ln f_{\mathrm{i}}$ is negative, so that $s$ is positive. The more narrow the mode of fig. 3 (i.e. the more ordered the state), the smaller $s$ becomes, so that in the limit of a zero thickness to the curve (meaning all the molecules are at a single speed i ) the limiting value of $s$ is zero. This shows that entropy is small in ordered states which are near equilibrium.

\section{Combined entropy changes?}

Prigogine [10] and others have proposed the addition of other entropies which could feed negative entropy into a given (open system). Consequently the total entropy is considered to be

$$
d s=d s_{T}+d s_{\text {logical }}
$$

where $d s$ is the total change in entropy, $d s_{T}$ is the change in thermodynamic entropy and $d s_{\text {logical }}$ is the change in entropy due to complexity - that is Prigogine or logical entropy. The thermodynamic entropy $d_{T}$ for a gas would be described by the Boltzmann law of eqn. (1), and for other types of energy exchange there will be an appropriate way of describing the internal energy, whether it be for electromagnetic, thermal, kinetic etc. While $d s_{T}$ tends to increase, the term $d s_{\text {logical }}$ can increase or decrease or remain zero (it is considered positive if entropy enters the system and negative if entropy leaves the system). The important implication of the additional logical entropy term is that then the total entropy change of any open system, $d s$, can be considered positive, negative, or zero. Systems for which $d s<0$ (that is where entropy is decreasing) are said to be self-organizing (Cambel [11]), though this term needs care since the organising is only reflecting an ordering principle already present.

A good example of $d s_{\text {logical }}$ would be the order inherent in crystals due to the atomic structure of a particular chemical compound. When such a compound is cooled to produce crystals, it is not the cooling itself which causes the crystals to occur, but the response to the precise molecular bonding within the material itself, and which is a definite function of the state variables. Often this is falsely used as an argument for increase in order (and thus an argument for increase in order) when in fact the ordering principle is latently already in the elements involved.

The all important question that many have addressed is how to quantify $d s_{\text {logical }}$ for real systems, particularly in the life sciences. It has been suggested with some cogency that, on the basis of Shannon's theory of information transmission ${ }^{3}$, one can express $d s_{\text {logical }}$ as an equivalent to the Boltzmann law of eqn. (1). This follows since Shannon's theory is based simply on parcelling any information into a series of irreducible packets such that at the fundamental level, a digital switch is either 'on' or 'off'. Each of these represents a state (rather like the discussion of molecule states in Boltzmann's theory in section 2.3) and adding up all the probabilities of whether each state is present, gives

$$
d s_{\text {logical }}=-L \sum_{i=1}^{N} p_{i} \ln p_{i}
$$


where $L$ is a constant whose value is of course one of the major difficulties, since the number of possible states of any open system is not known (particularly as quantum states may also need to be invoked). Setting this at unity is often the assumption in recent studies [12,13], but when dealing with arrangements of biological systems (such as the arrangement of DNA and the nucleotides, enzymes, ATP etc) the definition of what to include as a system state is moot. Thus Peter Coveney and Roger Highfield were being brutally honest when they stated in their classic book "The Arrow of Time" that [14]

"There is, however, nothing to tell us how fine the [parcelling] should be.

Entropies calculated in this way depend on the size-scale decided upon, in direct contradiction with thermodynamics in which entropy changes are fully objective"

There is another major difficulty which concerns the definition of information. Gitt [15] has shown that the Shannon information concept is not really the main contributor, since this carries no concept of function [7] and purpose (termed 'apobetics' in Gitt's work) which is essential to any real information exchange in any working system. Consequently to define complexity as a gradual seepage in of 'negative entropy' is predicated on the notion that information can gradually increase from a random state. However in reality information is not defined in the coded sequence itself (such as the DNA nucleotide sequence of fig. 1) but rather (Gitt has shown) as five levels of signal statistics: (the Shannon level), code (syntax), expression (i.e. message at the semantic level), expected action (pragmatics) and intended result (apobetics). To summarise just two of these levels succinctly, the code used is not defined by the material it orders, and the expression (message) is not defined by the code it uses. Gitt argues that information has to be thought of as a third fundamental quantity which cannot be defined in terms of matter and energy.

\section{A new approach: entropy constrained by functional information}

We propose a different treatment which quantifies the effect of functional information in a system. This approach recognises Gitt's important deductions concerning real information systems being impossible to define in terms of matter and energy alone. However one can recognise the effect of machines / information systems (that is teleonomy) being present in exactly the same way as a digitally controlled machine (i.e. a computer) is operated by software. The high level program controls a set of electronic switches on a micro chip which are set in a certain predefined pattern. Thus the logical entropy $d s_{\text {logical }}$ (the switching of the micro chip in the analogy) rather than being the source of the information should be thought of as the effect of information carrying systems. For a pure materialist there may be a natural reticence to adopting such an approach, but the evidence of the thermodynamics of living systems supports this. 


\subsection{Gibbs free energy}

An illustration of how an information bearing system relates to thermodynamic entropy is demonstrated by the code carrying DNA polymer (see fig. 1). As is well known, DNA is a double helix. The outer edges are formed of alternating ribose sugar molecules and phosphate groups. The two strands go in opposite directions either side of the nitrogenous bases which are like the inside rungs of a ladder. Adenine (A) on one side pairs with thymine (T), and on the other by hydrogen bonding, and cytosine $(\mathrm{C})$ pairs with guanine $(\mathrm{G})$. It has been noted that the C-G pair has three hydrogen bonds while the A-T pair has only two, which keeps them from pairing incorrectly. But this only dictates side-to-side pairing, but says nothing about the order along the molecule which is of course the all important digital information. There is no physical / chemical law which of itself stops other bonds forming which are not recognised in the DNA code, such as A-G or T-C though in terms of efficient use of space, the base pair A-T is identical in size to G-C which makes stacking very regular and precise. The point here is that it is the information contained in the DNA itself which causes particular bonds to be made, not the chemistry itself. Furthermore if one takes a solution of adenosine monophosphate (AMP) and a solution of thymidine monophsphate (TMP), and mix them together, they will not form base pairs A-T in solution because the bases will H-bond with water molecules. So this illustrates that for the information to exist at all in the system, there needs to be the correct thermodynamic energy relationships existing at the fundamental level, constrained by low levels of logical entropy (from high level information).

This is best discussed in the context of the Gibbs free energy $g$ which effectively takes away the unusable lost energy (associated with entropy) from the enthalpy $h$ (which can be regarded as the total thermodynamic energy available). Thus

$$
g=h-T s,
$$

It can be shown that for a chemical reaction, the change between the initial reactants to products is related to the change in the Gibbs free energy through

$$
\Delta g=-\frac{R T}{W} \ln K \text {, i.e. } K=-\exp (-W \Delta g / R T)
$$

where $K$ is the reaction rate constant. Assuming that the reaction itself proceeds at constant temperature, then from equation (4) one can also state that

$$
\Delta g=\Delta h-T \Delta s,
$$

and referring to base states (superscript 0 ) we have from equations (5a) and (6)

$$
\ln K=\frac{-W \Delta h^{0}}{R T}+\frac{W \Delta s^{0}}{R} \text {. }
$$

From eqn. (5b), for a reactant $F$ going to product $P$, the probability $p$ of any one state is given by

$$
p=\frac{K}{1+K}=\frac{\exp (-W \Delta g / R T)}{1+\exp (-W \Delta g / R T)}
$$

The equilibrium constant $K$ governs the progress of the chemical reaction to completion. The $K$ will be large where reactions have a maximum value of $\Delta s^{0}$ 
and a minimum value of $\Delta h^{0}$. Natural systems will tend to configurations where the entropy $\Delta s^{0}$ is greatest and the heat content $\Delta h^{0}$ is lowest. And we note that the lowest heat content configurations are generally associated with molecular configurations in which the atoms are bound most securely to one another. All chemical reactions without external influences will minimize $g$. Furthermore any natural process occurs spontaneously if and only if the associated change in Gibbs free energy $g$ for the system is negative $(\Delta g<0)$. Likewise, a system reaches equilibrium when the associated change in $g$ for the system is zero $(\Delta g=$ $0-$ and note that the probability $p$ is then $1 / 2$ ), and no spontaneous process will occur if the change in $g$ is positive $(\Delta g>0)$. It is the information within the structure which enables a non-equilibrium chemistry to be maintained, such that low logical entropy $\left(\Delta s_{\text {logical }}\right)$ is added to the fundamental molecular structure. Another very clear example is the famous Urey-Miller experiment which produced left handed and right handed chirality amino acids by firing sparks across a reducing mixture of methane, ammonia, water and hydrogen. The mixture was racemic in left handed and right handed chirality whereas in life systems one requires only left handed amino acids. The probability of any one state is in fact $1 / 2$ since there is equilibrium between the two possible end states. Only by driving the net Gibbs free energy between the two end states to an impossible infinite value (that is impossible without an information-rich machine) could one get an entirely left handed system which is what life systems actually do have. However if we consider the information in the system as being the source and the logical entropy as being the effect, then there is a logical coherency in the argument. (In this case from eqn. (6), $\Delta g$ is large and positive precisely because $\Delta s_{\text {logical }}$ is large and negative).

Consequently to suggest that reactions on their own can be moved against the free energy principle is not true, since they could not be sustained. The DNA molecule along with all the nucleotides and other polymers could not change radically such that a low entropy situation would emerge. To alter the DNA constituents from one stable state say to another representative state with a distinct improvement cannot be done by natural means alone without additional information. The thermodynamic laws are against such a procedure.

Dickerson hoped for a different physics when he stated [16]

"Through some gradual means, about which we can only speculate, an association of nucleic acids as the archival material with protein as the working catalyst evolved into the complex genetic transcription and translation machinery that all forms of life exhibit today"

\section{Conclusions}

In this paper we have considered the concept of logical entropy as a parallel to the Boltzmann probability formula for system states. We have then considered the role of information in reducing at a fundamental level the logical entropy and concluded that rather than regarding negative entropy as being a source of information at the fundamental level, it is far more self-consistent to regard the 
information defined in terms of a source from which negative logical entropy is derived at the molecular level, and which can be quantified using Shannon principles.

It has often been asserted that the logical entropy of an open system could reduce through chance exchanges of that system with its environment. By considering the Gibbs free energy connecting two possible states, it is evident that this involves thermodynamic hurdles which demand effectively a different physics. Self-organisation (so called) only takes place when existing information is already inherent in the system and not vice versa. In an open system, energy (such as from the sun) may increase the local temperature difference (and thus increase the potential for useful work that can be done locally), but without a machine (that is, a device which is made or programmed to use the available energy), there is still no possibility of the self-organisation of matter. There has to be previously written information or order (often termed "teleonomy") for passive, non-living chemicals to respond and become active. Thus the following summary statement applies to all known systems:

$$
\begin{gathered}
\text { Energy }+ \text { Information } \rightarrow \text { Locally reduced entropy (Increase of order) } \\
\text { (or teleonomy) }
\end{gathered}
$$

with the corollary:

\section{Matter and Energy alone $\not \Rightarrow$ Decrease in Entropy}

Another way of saying this is that for an open system, energy must be directed to be of any use.

In this paper we have argued that for living systems, rather than regarding negative entropy as a quantity generated within, one should regard the information as being the cause and the logical entropy reduction being the result. That which is dead (such as a stick or leaf from a tree) has no information or teleonomy within it to convert the sun's energy to useful work. Indeed it will simply heat up and entropy will increase. However, a living plant has information within it, such that the energy from the sun is absorbed (along with carbon dioxide and water) by its leaves, through photosynthesis. The chlorophyll of the leaf enables such a biochemical reaction to take place. To quote WilderSmith [17, p.59],

“..raw matter within a closed system, plus a teleonomic machine, might yield auto-organisation derived from endogenous [that which comes from within] energy. Raw matter within an open system, plus a teleonomic machine may yield auto-organisation derived from endogenous and/or exogenous [that which comes from without] energy. Within both open and closed systems, however, a mechanism (machine, teleonomy, know-how) is essential if any auto-organisation is to result." 


\section{References}

[1] Watson, J. D. and Crick, F. H. C. "Molecular structure of Nucleic Acids", Nature 171, 737-738 (1953).

[2] See for example Nicolis, G. and I. Prigogine. Exploring Complexity: An Introduction, Freeman, New York, (1989) and the useful web site where these matters of non-equilibrium thermodynamics are discussed: http://www.schuelers.com/ChaosPsyche/part_1_9.htm (accessed 2006).

[3] Shannon, C.E., "The Mathematical Theory of Communication", The Bell System Technical Journal, Vol. 27, pp. 379-423, 623-656, July, October, (1948).

[4] Dawkins, R. "The Information Challenge", pp. 107-122 (quote is from pp. 120-121), Chapter 2.3 of A Devil's Chaplain; Selected Essays by Richard Dawkins, Ed. Latha Menon, Phoenix, 2003.

[5] Jackson, S.P., "Sensing and repairing DNA double strand breaks", Carcinogenesis, Vol. 23, No. 5, 687-696, OUP, May 2002.

[6] de Laat W. L., Jaspers, N.G.J., and Hoeijmakers, J.H.J., "Molecular mechanism of nucleotide excision repair", Genes and Development, Vol. 13, No. 7, pp. 768-785, April, 1999.

[7] Meyer, S.C., "The origin of biological information and the higher taxonomic categories", Proceedings of the Biological Society of Washington, 117(2), 213-239, 2004.

[8] Conway Morris, S., "Evolution : bringing molecules into the fold", Cell 100, 1-11, 2000.

[9] Klyce, B. "The second law of thermodynamics", essay at http://www.panspermia.org/seconlaw.htm (accessed 2006).

[10] Nicolis G. and Prigogine, I., Exploring complexity: An introduction, W.H. Freeman, New York, 1989.

[11] Çambel, A. B., Applied chaos theory: A paradigm for complexity, Academic Press, Boston: 1993.

[12] Wicken, J.S., Evolution, Thermodynamics and Information: Extending the Darwinian Program, Oxford University Press, 1987.

[13] Penrose, R., The Emperor's New Mind, Oxford University Press, 1989.

[14] Coveney, P. and Highfield, R., The Arrow of Time, Ballentine Books, 1990. p 176-177.

[15] Gitt, W, "Information: the third fundamental quantity", Siemens Review, Vol 56, Part 6, pp.36-41, 1989.

[16] Dickerson, R.E. "Chemical Evolution and the origin of life", Scientific American 239(9), 73, 1978.

[17] Wilder-Smith, A.E., "The Natural Sciences know nothing of evolution", Master Books, San Diego, California 1981. See particularly chapter 4 "The Genesis of Biological Information". Bracketed material added. 